\title{
Clear Experimental Demonstration of Hole Gas Accumulation in Ge/Si Core-Shell Nanowires
}

Naoki Fukata, ${ }^{*}$ Mingke Yu, $†$ Wipakorn Jevasuwan, $\dagger$ Toshiaki Takei, $\uparrow$ Yoshio Bando, $\uparrow$ Wenzhuo $W u$, $t$ and Zhong Lin Wangt, $†$

$广$ International Center for Materials Nanoarchitectonics, National Institute for Materials Science, 1-1 Namiki, Tsukuba, 305-0044, Japan

$¥$ School of Materials Science and Engineering, Georgia Institute of Technology, Atlanta, GA 30332-0245, USA

\section{Detailed TEM images of Ge/Si core-shell nanowires}

The shell crystallinity and the interface in $\mathrm{Ge} / \mathrm{Si}$ core-shell nanowires are important. The interface between Ge and Si should be sharp and free of defects. Figures 1 and 4 show typical TEM images. We also obtained large, clear images of Ge/Si core-shell NWs. Figure S1 shows high-magnification TEM images of $\mathrm{Ge} / \mathrm{Si}$ core-shell nanowires $\left(\mathrm{B}_{2} \mathrm{H}_{6}\right.$ flux: $0.5 \mathrm{sccm}$, shell growth time: $\left.30 \mathrm{sec}\right)$. The i-Ge core and the p-Si shell can be clearly distinguished in Figure S1 (a). The p-Si shell layer is a complete single crystal. No defects can be observed in the interface between the i-Ge core and the p-Si shell
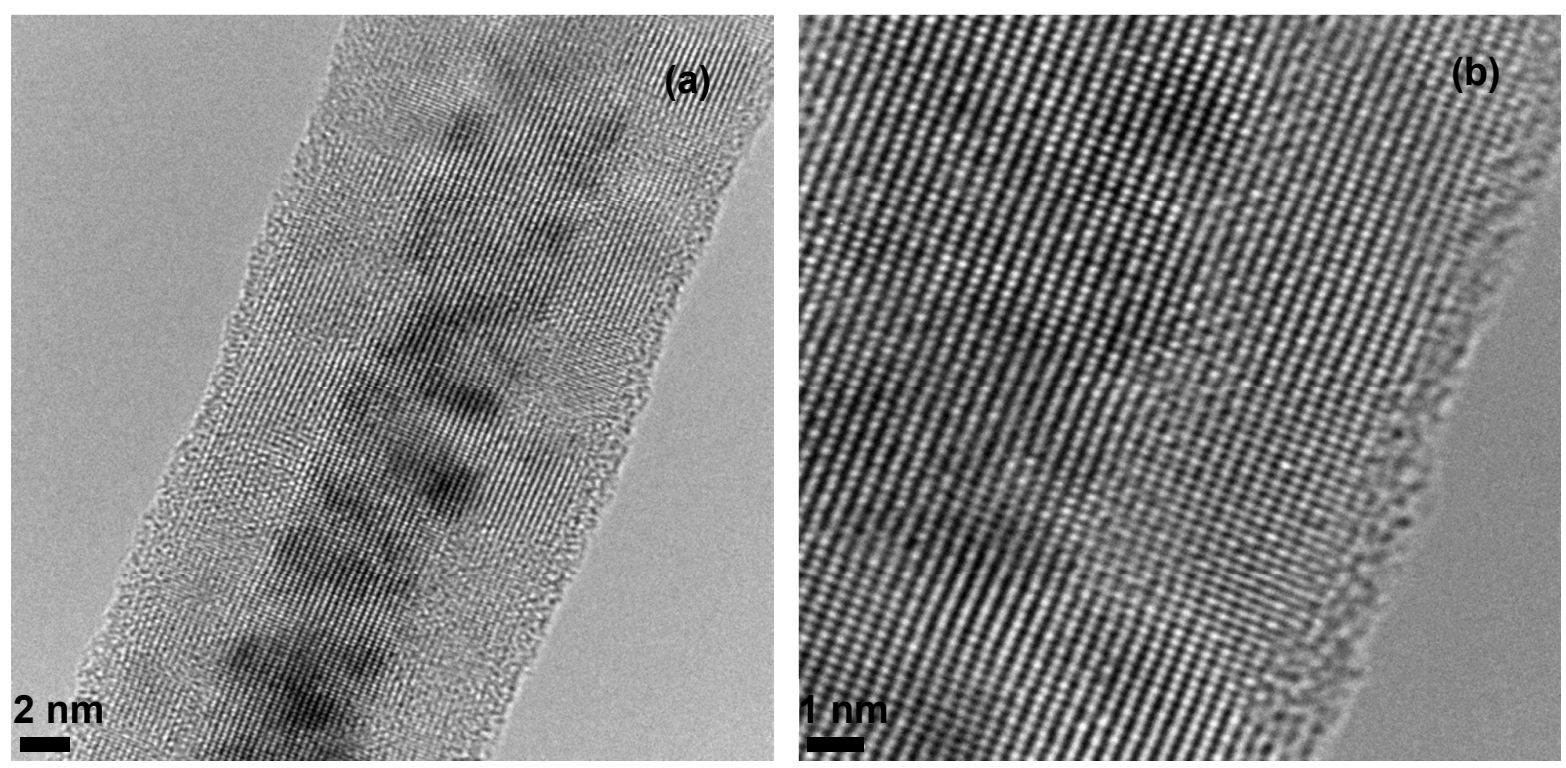

Figure S1. TEM images of i-Ge/p-Si core-shell NWs with a shell growth time of $30 \mathrm{sec}$ and $\mathrm{B}_{2} \mathrm{H}_{6}$ gas flux of $0.5 \mathrm{sccm}$. (a) Low-resolution and (b) high-resolution images. 
shown in Figure S1 (b). Figure S2 shows a TEM image of i-Ge/p-Si core-shell NWs with a shell growth time of 1 min and a $\mathrm{B}_{2} \mathrm{H}_{6}$ gas flux of $0.5 \mathrm{sccm}$. We can see both polycrystalline structures (Figure S2 (a)) and single-crystal structures (Figure S2 (b)) in the p-Si shell layers. The crystallinity of the $\mathrm{p}$-Si shell region closely depends on the thickness. The p-Si shell layer can adopt a single-crystal structure if its thickness is less than $5 \mathrm{~nm}$.
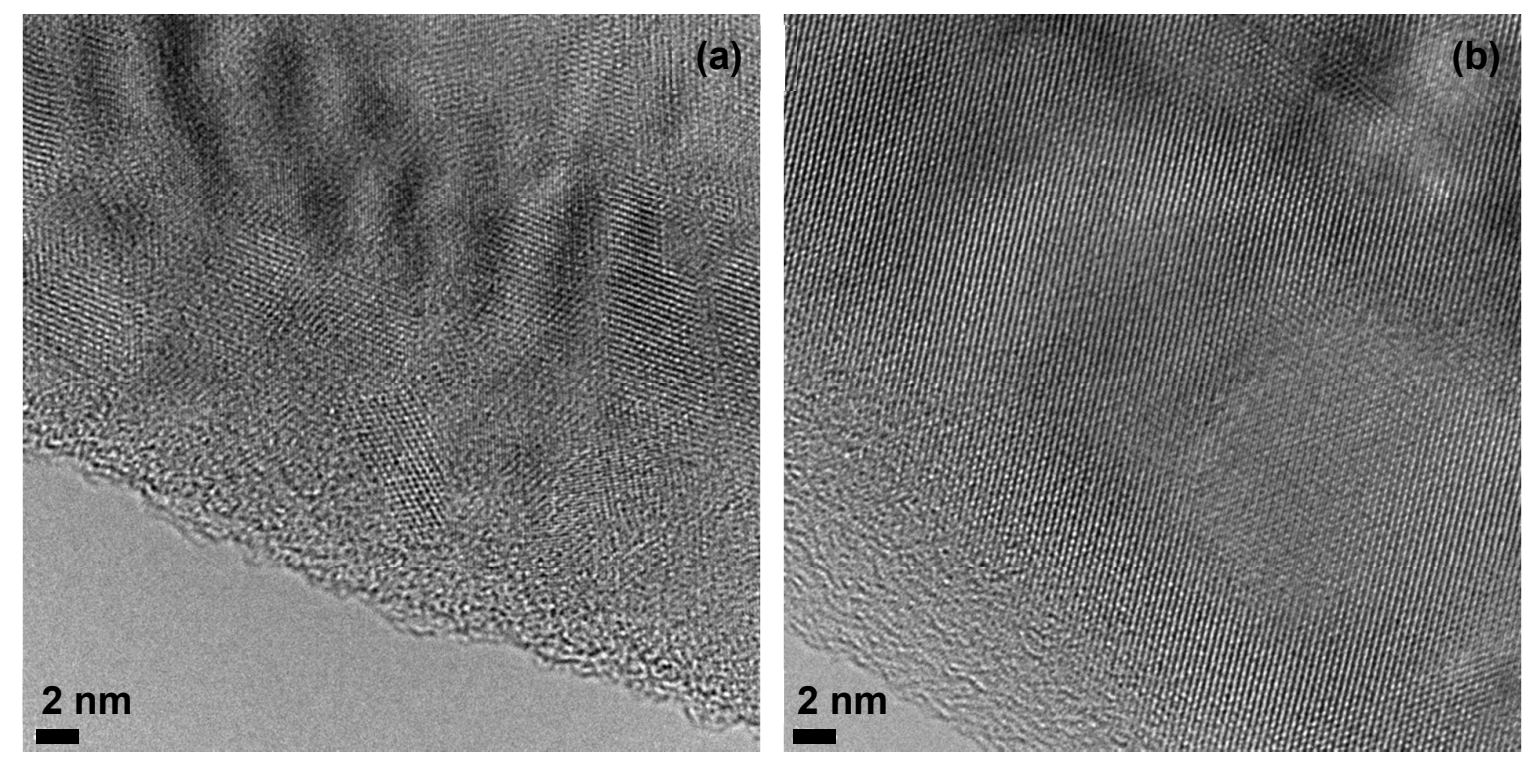

Figure S2. TEM images of p-Si shell layers in i-Ge/p-Si core-shell NWs with a shell growth time of $1 \mathrm{~min}$ and $\mathrm{B}_{2} \mathrm{H}_{6}$ gas flux of $0.5 \mathrm{sccm}$. Images (a) and (b) show polycrystal and single-crystal regions, respectively.

\section{Peak shift of the Si and Ge optical phonon peaks as a function of shell growth time}

In Figure 2, we show only typical Si and Ge optical phonon peaks. Their peak shifts as a function of shell growth time and $\mathrm{B}_{2} \mathrm{H}_{6}$ gas flux. The peak shift and shape of optical phonon peaks includes important information on the stress in the core and shell regions and the electrical activation of dopant atoms related to the Fano effect. As further evidence of this dependence, Figure S3 shows more detailed data. The intensity of the Si optical phonon peak increased on lengthening the shell growth time, and the peak showed asymmetric broadening due to the Fano effect. This result clearly demonstrates the electrical activation of $\mathrm{B}$ atoms in the $\mathrm{Si}$ shell layers, giving evidence of the formation of p-type Si shell layers. On the other hand, the peak intensity of the Ge optical phonon peak decreased on lengthening the shell growth time. The Raman scattering from the Ge core region is disturbed by the Si shell layers, because the Ge region is in the deeper core region of core-shell NWs. All the data related to the Ge and Si optical phonon peak in the i-Ge/p-Si core-shell NWs are summarized in Figure S3 (b) and (c). The dependences shown in Figure S3 (b) and (c) are similar to those shown in Figure 2 (c) and (d). The downshift of the Si optical phonon peak for a shell growth 
time of $30 \mathrm{sec}$ is mainly due to the tensile stress from the Ge core region. The Si optical phonon peak shows a further downshift with longer shell growth time. This can be explained by the Fano effect and stress relaxation. The Ge optical phonon peak shows the upshift in short shell growth times, which is due to the compressive stress applied by the Si shell. The Ge optical phonon peak then shows a downshift with longer shell growth time. This can be explained by stress relaxation and hole accumulation. All core-shell NWs show the same pattern.
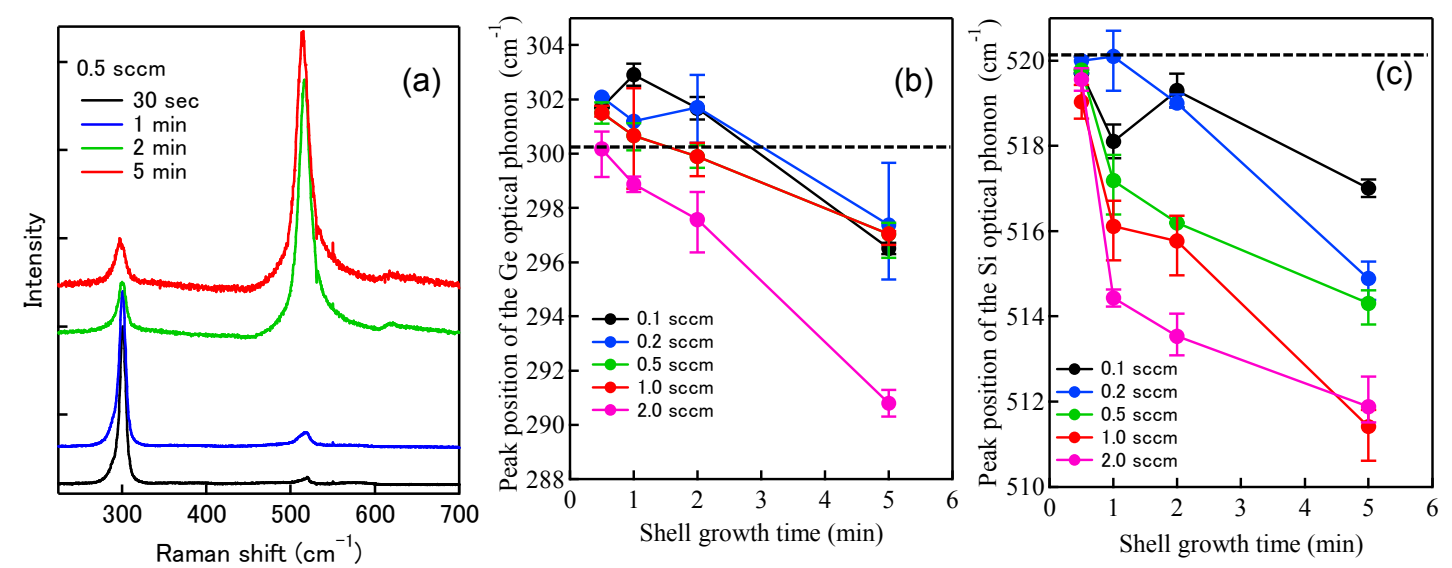

Figure S3. (a) Dependence of Raman spectra observed for $\mathrm{i}-\mathrm{Ge} / \mathrm{p}-\mathrm{Si}$ core-shell NWs on shell growth time. The $\mathrm{B}_{2} \mathrm{H}_{6}$ gas flux was set at $0.5 \mathrm{sccm}$. Raman shift of (b) Ge and (c) Si optical phonon peaks as a function of shell growth time. The $\mathrm{B}_{2} \mathrm{H}_{6}$ gas flux was changed from $0.1 \mathrm{sccm}$ to $2.0 \mathrm{sccm}$. The dotted lines are the peak positions of the optical phonon peak for bulk Ge and Si.

\section{Characterization by XRD}

Figure S4 shows an XRD pattern observed for i-Ge/p-Si core-shell NWs. A scanning rate of $0.5 \mathrm{~s}^{-1}$ was applied to record clear XRD patterns. In addition to the Ge (111) and Si (111) peaks, we observed small peaks related to $\mathrm{Ge}$ (220), $\mathrm{Si}$ (220), Ge (311), Si (311) Ge (511) and $\mathrm{Si}$ (511) peaks. The average lattice constants of the Ge core and Si shell were calculated by fitting the XRD peaks. Figure S5 shows typical Ge (111) and Si (111) XRD peaks observed for i-Ge/p-Si $(0.5 \mathrm{sccm})$. The dotted lines are deconvoluted peaks acquired by fitting that estimate the peak position of the Ge (111) and $\mathrm{Si}$ (111) XRD peaks. The Ge (111) peak shifts to a lower angle, while the Si (111) peak shifts to a higher angle on lengthening the $\mathrm{Si}$ shell growth time. The result shows the same pattern as $\mathrm{i}-\mathrm{Ge} / \mathrm{p}-\mathrm{Si}(0.1 \mathrm{sccm})$ shown in Figure 3 (a). 


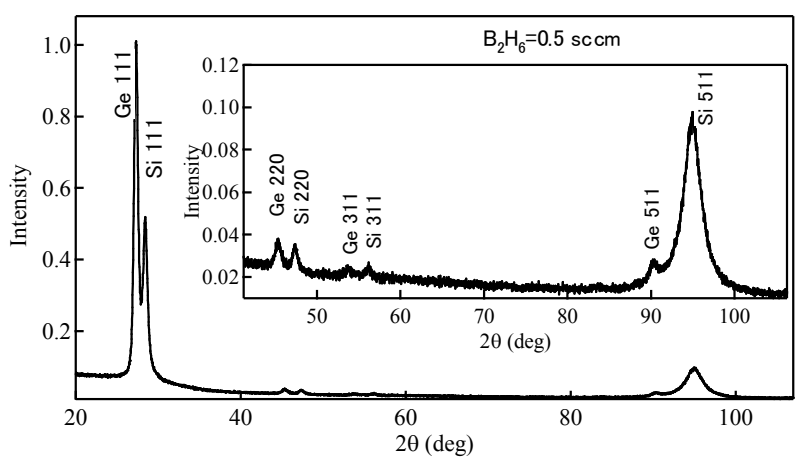

Figure S4. XRD spectra observed for i-Ge/p-Si core-shell NWs.

\section{Effect of HF etching}

To further confirm hole accumulation in the Ge core

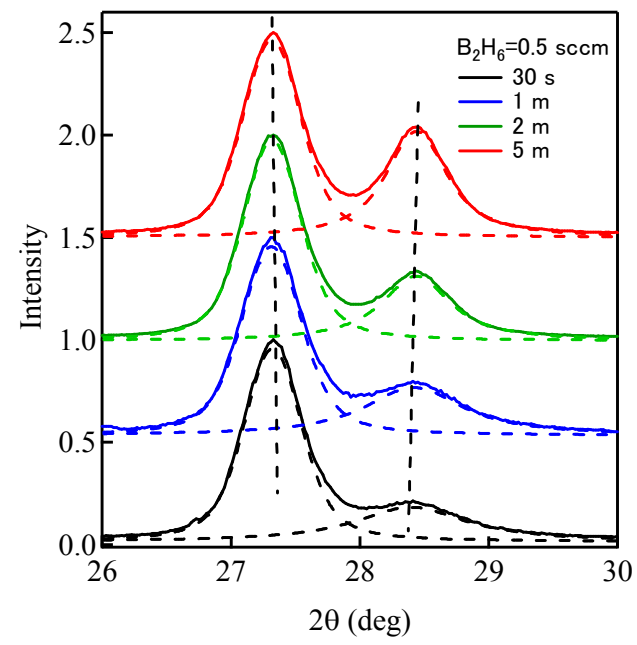

Figure S5. XRD spectra of Ge (111) and $\mathrm{Si}(111)$ observed for $\mathrm{i}-\mathrm{Ge} / \mathrm{p}-\mathrm{Si}$ core-shell NWs.

region, the Si shell layer was etched with HF solution. Figure S6 shows the Ge optical phonon peak before and after HF etching. The Ge optical phonon peak clearly shows asymmetric broadening due to the Fano effect before HF etching. After HF etching, the asymmetric broadening has decreased and the peak has shifted to a higher wavenumber. This cannot be explained by any change in stress applied by the $\mathrm{p}$-Si shell layers, since the Si shell layer is already relaxed due to the introduction of dislocations, as explained in Figures 2 and 4 above. The thickness of B-doped Si shell layers decreases as a result of HF etching, so the number of holes introduced into the Ge core region is likely to have decreased, resulting in a decrease in hole accumulation in the Ge core region. Hence, the result of Figure S5 also indicates the asymmetric broadening to be most likely due to hole accumulation from the $\mathrm{p}-\mathrm{Si}$ shell to the $\mathrm{i}-\mathrm{Ge}$ core region.

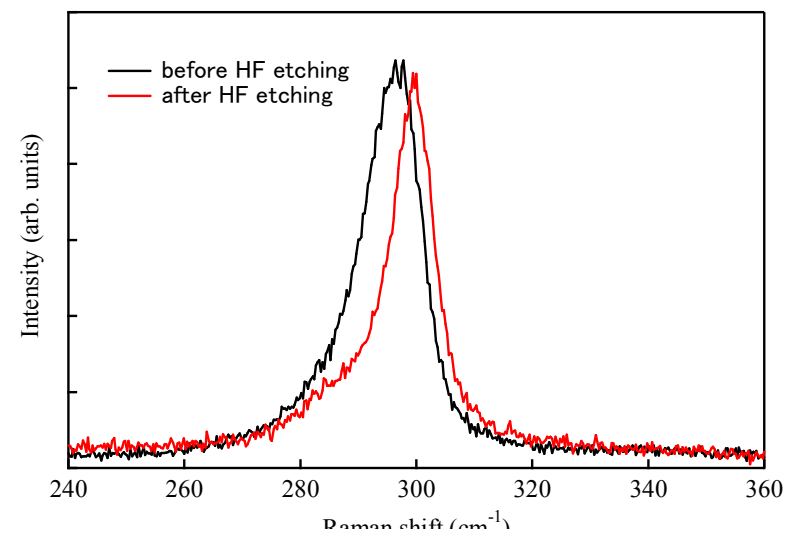

Figure S6. Ge optical phonon peaks observed for $\mathrm{i}-\mathrm{Ge} / \mathrm{p}-\mathrm{Si}$ core-shell NWs before and after HF etching. 